\title{
Performances during the grazing season in Pyrenean conditions
}

\author{
I. Casasús, D. Villalba, R. Ferrer, A. Sanz, R. Revilla \\ SIA-DGA, Apdo. 727, 50080 Zaragoza, Spain
}

The performances of a spring-calving Parda Alpina and Pirenaica suckler herd and an autumn-calving Parda Alpina suckler herd on high mountain and forest ranges over eight grazing seasons (1988-1996) were analysed. Only data from the cows that calved in two consecutive years before and after a grazing season were considered, i.e., 196 cycles for autumn-calving and 356 cycles for springcalving cows. Cows suckled their calves for 5-6 months, which is the traditional management in the Spanish Pyrenean mountains. Multiparous autumn-calving cows gained $55.2(2.2) \mathrm{kg}$ over the 158-day grazing season, while Parda Alpina spring-calving cows gained 13.5 (2.7) kg and Pirenaica cows gained $20.6(2.8) \mathrm{kg}$ in 183 days. These suckled their calves for the first 100 days of the grazing season, and average daily gains at pasture were 838 (14) and 809 (11) g in Parda Alpina and Pirenaica calves. Year, parity, initial live weight and breed significantly affected performances at pasture. Primiparous cows had lower gains than multiparous cows $(P<0.001): 41.4(5.4)$ and $1.1(4.3) \mathrm{kg}$ for autumn- and spring-calving cows respectively. These results show the inability of Pyrenean mountain pastures to allow for important weight gains during the grazing season. Grazing contributed for slightly over $40 \%$ of the annual net energy intake, whatever the calving period and the breed. This stresses the need to design yearlong management practices consistent with the extensification of this system.

\section{Adaptation des techniques GPS à l'étude de la localisation d'herbivores domes- tiques au pâturage}

\author{
F. Decuq ${ }^{\text {a }}$ J.-P. Brun ${ }^{\text {b }}$ H. Dubroeucq ${ }^{\mathrm{a}}$, M. Thériez ${ }^{\mathrm{a}}$, D. Micol $^{\mathrm{a}}$
}

${ }^{a}$ Laboratoire adaptation des herbivores aux milieux $;{ }^{b}$ Département élevage et nutrition des animaux, Inra, 63122 Saint-Genès-Champanelle, France

Le suivi de la localisation sur des surfaces de pâturage est difficile et lourd en moyens et temps d'observation. Nous avons développé une méthode de suivi automatique et autonome à partir du système satellitaire GPS (Global Positioning System) avec des récepteurs portés par les animaux. Ce dispositif, permet de définir la position des animaux (longitude, latitude, altitude). La précision globale de la méthode est améliorée par l'utilisation du mode différentiel de GPS reposant sur la présence d'un récepteur fixe permettant de corriger le brouillage induit volontairement pour l'utilisation civile du système. Ainsi, nous avons pu obtenir une précision des itinéraires en continu de $\pm 1,5 \mathrm{~m}$ pour des bovins ou des chevaux exploitant une estive de 80 ha en moyenne montagne ( $1000 \mathrm{~m}$ ). L'autonomie électrique et la capacité de mémorisation des récepteurs (un enregistrement toutes les 5 secondes), permettent un fonctionnement continu de $30 \mathrm{~h}$. L'équipement (récepteur, batterie, support) pèse $6 \mathrm{~kg}$ et ne semble pas gêner les sujets équipés. Cette méthode de localisation des animaux est globalement efficace, puisque les enregistrements couvrent $91,5 \%$ du temps total d'observation. Chaque jour de mesure, plus de 15500 localisations sont enregistrées par animal et permettent de reconstituer son itinéraire. Ainsi, nous avons pu estimer les distances parcourues quotidiennement par les chevaux ( $10 \mathrm{~km}$ en moyenne) et les bovins $(6 \mathrm{~km})$. 